\title{
Necessidades humanas básicas e dependência de cuidados de enfermagem de idosos institucionalizados
}

\author{
Basic human needs and nursing care dependency of institutionalized elders
}

Necesidades humanas básicas y dependencia de cuidados de enfermería en ancianos institucionalizados

\section{Luanna dos Santos Rocha ${ }^{1}$, Elizabeth Moura Soares de Souza², Célia Alves Rozendo ${ }^{3}$}

\author{
${ }^{1}$ Enfermeria. Discente do Programa de Pós-Graduação em Enfermagem da Universidade Federal de Alagoas (UFAL). Maceió, AL, Brasil. E-mail: \\ luanna.rocha.enf@gmail.com. \\ ${ }^{2}$ Enfermeira. Discente do Programa Interunidades de Doutoramento em Enfermagem da Universidade de São Paulo. Professora Assistente Escola de \\ Enfermagem e Farmácia (ESENFAR) da UFAL. Maceió, AL, Brasil. E-mail: elmososo@gmail.com. \\ ${ }^{3}$ Enfermeira, Doutora em Enfermagem. Professora Adjundo da ESENFAR/UFAL. Maceió, AL, Brasil. E-mail: celia.rozendo@gmail.com.
}

\section{RESUMO}

Esta pesquisa objetivou identificar as necessidades humanas básicas e o grau de dependência de cuidados de enfermagem de idosos institucionalizados. Trata-se de estudo quantitativo descritivo observacional realizado com 67 idosos residentes em uma Instituição de Longa Permanência para Idosos, de Maceió, AL. Utilizou-se para coleta de dados instrumento contendo dados de caracterização da Instituição e dos sujeitos e o Sistema de Classificação de Pacientes. Os resultados apontam o predomínio de idosos acima dos 70 anos, sexo masculino, não alfabetizados, solteiros, residentes a aproximadamente 5,5 anos na instituição. As necessidades humanas básicas mais afetadas eram: cuidado corporal, educação à saúde, locomoção, eliminações e mobilidade. O grau de dependência dos cuidados de enfermagem mais observado foi a dependência parcial. Os resultados permitem tanto uma melhor compreensão das reais necessidades demandadas por idosos institucionalizados, quanto colaborar para a (re)organização do serviço de enfermagem.

Descritores: Idoso; Enfermagem Geriátrica; Instituição de Longa Permanência para Idosos.

\section{ABSTRACT}

The objective of this study was to identify the basic human needs and the level of dependency on nursing care presented by institutionalized elders. This observational, quantitative and descriptive study was performed with 67 elder inpatients of a Geriatric Long-Term Care Institution in Maceió, Alagoas, Brazil. Data collection was performed using an instrument containing data regarding the institution's and subjects' characteristics as well as the Patient Classification System. Results show a predominance of male patients, older than 70 years, illiterate, single, and living in the institution for approximately 5.5 years. The most affected basic human needs were: body care, health education, locomotion, elimination, and mobility. A degree of partial dependency on nursing care was the most commonly observed. Results permit a better understanding of the true needs of institutionalized elders and collaborate with the (re)organization of nursing care.

Descriptors: Aged; Geriatric Nursing; Homes for the Aged.

\section{RESUMEN}

Este estudio objetivó identificar las necesidades humanas básicas y el grado de dependencia de cuidados de enfermería por parte de ancianos institucionalizados. Estudio cuantitativo, descriptivo, observacional, realizado con 67 ancianos residentes en una Institución de Larga Estancia para Ancianos de Maceió-AL. Datos recolectados mediante instrumento conteniendo información de caracterización de la Institución y de los sujetos y el Sistema de Clasificación de Pacientes. Los resultados expresan predominio de ancianos mayores de 70 años, sexo masculino, no alfabetizados, solteros, con estancia promedio de 5,5 años en la institución. Las necesidades humanas básicas más afectadas eran: cuidado corporal, educación de salud, locomoción, eliminaciones y movilidad. El grado de dependencia de cuidados de enfermería más observado fue la dependencia parcial. Los resultados permiten tanto una mejor comprensión de las reales necesidades demandadas por los ancianos institucionalizados como colaborar para la (re)organización del servicio de enfermería. Descriptores: Anciano; Enfermería Geriátrica; Hogares para Ancianos. 


\section{INTRODUÇÃO}

O Brasil vem experimentando o crescimento e longevidade de sua população. Levantamentos estatísticos realizados pelo Instituto Brasileiro de Geografia e Estatística (IBGE) no ano 2010 mostram que 10,8\% da população brasileira possuía 60 anos ou mais aproximadamente 20.590.599 idosos, sendo 9.156 .112 $(44,5 \%)$ homens e 11.434 .487 (55,5\%) mulheres. Ainda segundo 0 IBGE 0 estado de Alagoas possui aproximadamente 276.763 idosos, equivalente a cerca de $8,9 \%$ da população alagoana, dos quais 123.804 (44,7\%) são do sexo masculino e 152.959 (55,3\%) do sexo feminino $^{(1)}$.

Esse processo de envelhecimento populacional se reflete como um dos maiores desafios para a saúde pública contemporânea, especialmente nos países em desenvolvimento, onde a pobreza e a desigualdade social ganham destaque. No entanto, cabe a ressalva que esta transição demográfica reflete ganhos para o Estado e para a sociedade, visto que se apresenta como um produto da redução da fecundidade, da mortalidade infantil e da mortalidade nas idades mais avançadas(2).

O cuidado a pessoa idosa perpassa a força de vontade de um indivíduo ou da estrutura familiar, que idealmente deve se responsabilizar pela saúde e bem-estar do idoso; ele inclui uma rede social, infra-estrutura, recursos materiais apropriados, pessoal qualificado, apoio social, entre outras.

Quando existe uma lacuna aberta pela impossibilidade da família atender às necessidades de seus idosos, seja pela falta de condições socioeconômicas, que não permitam manter o seu ente no lar junto com a família, quer por exigências e incompatibilidades das sociedades atuais no que se refere à organização laboral e da família, quer pela falta de políticas públicas, que visem apoiar os idosos e seus familiares no cumprimento de seu papel, as instituições de longa permanência para idosos (ILPI) surgem a fim de preencher esse espaço. Essas instituições visam sempre complementar e nunca substituir a família(3).

Além disso, a institucionalização surge como alternativa quando a pessoa idosa sente-se sozinha, com medo da violência urbana ou vivencia uma exclusão familiar, emergindo a possibilidade do apoio, tanto no campo da saúde como no cuidado de si(4-5).

Embora se observe os esforços atuais no desenvolvimento de políticas públicas, sociais e de saúde dirigidas à população idosa, constata-se que o investimento em instalação de serviços gerontogeriátricos e na melhoria dos já existentes, não corre na mesma velocidade do crescimento da população idosa, do aumento da longevidade das pessoas e consequente fragilização(6).

Atualmente no estado de Alagoas, 508 idosos (0,2\% da população idosa do estado) encontram-se residindo em ILPI. Este número, embora pequeno, torna-se significativo quando relacionado à fragilidade apresentada por estes sujeitos e as situações que levaram à institucionalização, visto que entre as causas de institucionalização, estão a miséria, o abandono e as sequelas das doenças crônicas não transmissíveis ${ }^{(7)}$.

Assim, uma das funções mais essenciais destas instituições consiste na busca de recursos voltados à melhoria da qualidade de vida dos idosos que ali residem ${ }^{(8)}$. Para que uma ILPI possa promover a qualidade de vida dos seus residentes é preciso que esteja preparada e possua infraestrutura interna e externa adequadas, bom gerenciamento, recursos materiais adequados e suficientes, cumpra a política de aplicação e defesa dos direitos do idoso e possua uma equipe multiprofissional capacitada e disposta a atuar. A presença do enfermeiro dentro desta equipe é fundamental, refletindo diretamente na qualidade da assistência prestada às necessidades básicas apresentadas pelos idosos ${ }^{(9)}$.

Tais necessidades podem ser mais bem compreendidas com o conhecimento sobre a teoria das Necessidades Humanas Básicas que visa colaborar na determinação de prioridades de cuidado a uma pessoa, contribuindo para o planejamento de cuidados individualizados pelos enfermeiros, a uma determinada pessoa(10). Com essa teoria o enfermeiro utiliza prioridades para organizar o Processo de Enfermagem de acordo com as reais necessidades do sujeito.

Uma das formas que o enfermeiro gerontológico pode contribuir para a avaliação das necessidades dos sujeitos é por meio de sistemas de classificação de pacientes (SCP). Estes consistem em processos capazes de determinar, validar e monitorar as necessidades de cuidado dos pacientes, utilizando dados obtidos como subsídios para o dimensionamento de recursos humanos, planejamento de custos e qualidade da assistência (11).

Um dos sistemas adotado pela enfermagem рага a classificação dos clientes é o criado e validado com base 
no levantamento das necessidades humanas básicas individuais e de cuidado de enfermagem. Tal instrumento avalia 13 indicadores críticos de ordem biológica e psicossocial, a saber: estado mental e nível de consciência, oxigenação, sinais vitais, nutrição e hidratação, motilidade, locomoção, cuidado corporal, eliminações, terapêutica, educação à saúde, comportamento, comunicação e integridade cutâneo mucosa(11-12).

Cada um dos indicadores possui gradação (escore) de um a cinco, objetivando apontar a intensidade crescente de complexidade de cuidado. $O$ valor obtido individualmente em cada um dos indicadores é então somado e o total é comparado com pontuações existentes, conduzindo a uma classe ou categoria de cuidados a que este paciente pertence, a saber ${ }^{(11-12)}$ :

- Cuidados Mínimos: 13 a 26 pontos - cuidados a clientes estáveis sob o ponto de vista de enfermagem, mas fisicamente autossuficientes quanto à satisfação de suas necessidades humanas básicas.

- Cuidados Intermediários: 27 a 39 pontos. Cuidados a clientes estáveis sob o ponto de vista clínico e de enfermagem, com parcial dependência das ações de enfermagem para o atendimento de suas necessidades humanas básicas.

- Cuidados Semi-Intensivos: 40 a 52 pontos Cuidados a clientes crônicos, estáveis sob o ponto de vista clínico, porém, com total dependência das ações de enfermagem quanto ao atendimento das necessidades humanas básicas ou cuidados a pacientes recuperáveis (não crônicos).

- Cuidados Intensivos: 53 a 65 pontos - Cuidados a clientes graves, com risco iminente de vida, sujeitos à instabilidade de sinais vitais, que requeiram assistência de enfermagem permanente e especializada.

Ainda tomando como base este SCP pode se estabelecer uma relação entre o nível de dependência de enfermagem e os escores de cuidado dos indicadores avaliados, onde se classifica como independência no predomínio do escore um na avaliação dos indicadores, refletindo a capacidade de auto-cuidado; dependência parcial na presença dos escores dois, três e quatro na avaliação dos indicadores, refletindo que os cuidados de enfermagem podem situar-se em termos de ajuda, orientação, supervisão e encaminhamento; e dependência total no predomínio do escore cinco na avaliação dos indicadores refletindo que a enfermagem tem que fazer pelo cliente tudo aquilo que este não tem condições de fazer por si ${ }^{(11-12)}$.

Com base nas informações expostas o problema que encaminhou o desenvolvimento desta pesquisa foi: quais as necessidades humanas básicas mais afetadas e os graus de dependência de cuidados de enfermagem de idosos institucionalizados? Ao responder essa questão se objetiva identificar as necessidades humanas básicas e o grau de dependência de cuidados de enfermagem de idosos institucionalizados.

\section{METODOLOGIA}

Estudo de natureza quantitativa, descritiva observacional, realizado em uma ILPI de Maceió, escolhida por ser o local de referência para atividades práticas da disciplina de Saúde do Idoso do curso de graduação em enfermagem da Universidade Federal de Alagoas. Os sujeitos foram 67 idosos residentes na ILPI. Foi excluído desta população um residente por possuir menos de 60 anos, não se enquadrando aos critérios de inclusão que foram: possuir idade igual ou superior a 60 anos, concordar em participar da pesquisa mediante assinatura do Termo de Consentimento Livre e Esclarecido e residir na instituição (não importando o tempo de residência).

A pesquisa se iniciou após aprovação do Comitê de Ética em Pesquisa da UFAL, sob o Parecer Consubstanciado $N^{\circ}$ 004529/2011-12, de 26 de setembro de 2011. A coleta dos dados foi realizada nos meses de setembro e outubro de 2011, por meio de um roteiro préelaborado contendo dados de caracterização da ILPI (relativos à natureza, tempo de funcionamento, profissionais que compõem a equipe e número de residentes), de caracterização do sujeito (informações sobre idade, sexo, raça, estado civil, tempo de residência, escolaridade, profissão e aposentadoria) e o Sistema de Classificação de Pacientes (contendo os 13 domínios: estado mental e nível de consciência, oxigenação, sinais vitais, nutrição e hidratação, motilidade, locomoção, cuidado corporal, eliminações, terapêutica, educação a saúde, comportamento, comunicação e integridade cutâneo-mucosa). O roteiro foi preenchido pelas 
pesquisadoras por meio da técnica da observação sistemática e análise de prontuário.

Para a organização, tabulação e análise dos dados estatísticos descritivos utilizou-se o programa Microsoft Excel 2007. A análise descritiva ocorreu a partir de frequência absoluta (Fi) e percentual (F\%) e os resultados foram apresentados de forma descritiva, por meio de tabelas e quadros.

\section{RESULTADOS}

No período de estudo a população total da ILPI era de 68 residentes, dos quais apenas um não era idoso (sendo por isso excluído da população deste estudo). Formada em sua maioria por homens (59,9\%); a faixa etária predominante da população estava entre 71 e 75 anos $(25,4 \%)$, seguida dos sujeitos entre 76 e 80 anos (17,9\%) e entre 81 e 85 anos (14,9\%). Destaca-se a presença de cinco idosos com mais de 90 anos.

Quanto à raça dos sujeitos não se observa grande diferença entre negros, brancos e pardos, destacando-se o predomínio de negros e pardos sobre os brancos. No que trata ao estado civil observa-se que a maioria dos idosos são solteiros (58,2\%), existindo também na população idosos casados (16,4\%) e viúvos (16,4\%) - 9\% não souberam informar o estado civil. Quanto à escolaridade grande parte dos sujeitos, $47,8 \%$, não haviam sido alfabetizados, ao passo que $37,4 \%$ possuía o ensino fundamental, médio ou superior e $14,9 \%$ não souberam informar sua escolaridade.

No que diz respeito ao tempo de institucionalização constata-se que grande parte dos idosos residem na ILPI entre um e cinco anos (43,3\%), seguidos por aqueles que vivem de seis a 10 anos na instituição (20,9\%). A média de tempo de institucionalização é de 5,6 anos para homens e 5,4 anos para mulheres.

Analisando os dados referentes às necessidades humanas básicas afetadas, constatou-se que o tipo de cuidado de enfermagem mais exigido pelos idosos institucionalizados eram os cuidados mínimos, seguidos pelos cuidados intermediários e semi-intensivos, não existindo sujeitos em cuidados intensivos. (Tabela 1).

Tabela 1: Distribuição dos idosos residentes em ILPI segundo tipos de cuidados requeridos. Maceió-AL, 2011.

\begin{tabular}{ccc}
\hline Tipo de cuidado & Fi & F\% \\
\hline Cuidados mínimos & 38 & 56,7 \\
Cuidados intermediários & 25 & 37,3 \\
Cuidados semi-intensivos & 4 & 6,0 \\
Cuidados intensivos & 0 & 0,0 \\
\hline Total & 67 & 100 \\
\hline
\end{tabular}

Observa-se de forma mais detalhada que determinados escores dos indicadores se destacaram mais, colaborando para o aumento dos índices em cada nível de gradação segundo as necessidades básicas dos idosos. Os indicadores que sobressaíram neste estudo - e por isso serão descritos de forma mais minuciosa na sequência - foram: cuidado corporal, educação a saúde, locomoção, eliminações e motilidade. Ressalta-se que todos os 13 indicadores críticos foram avaliados durante a pesquisa. (Tabela 2 ).

Tabela 2: Distribuição dos indicadores segundo os escores de avaliação dos idosos residentes em ILPI. Maceió-AL, 2011.

\begin{tabular}{|c|c|c|c|c|c|}
\hline \multirow{2}{*}{ Indicadores } & \multicolumn{5}{|c|}{ Distribuição dos escores (F\%) } \\
\hline & 1 & 2 & 3 & 4 & 5 \\
\hline Cuidado corporal & 25,4 & 20,9 & 13,4 & 32,8 & 7,5 \\
\hline Educação a saúde & 29,9 & 29,9 & 19,4 & 11,9 & 8,9 \\
\hline Locomoção & 37,3 & 14,9 & 28,4 & 11,9 & 7,5 \\
\hline Eliminações & 35,8 & 9,0 & 46,2 & 9,0 & 0 \\
\hline Motilidade & 16,4 & 53,7 & 20,9 & 4,5 & 4,5 \\
\hline Estado mental ou nível de consciência & 35,8 & 29,9 & 17,9 & 16,4 & 0 \\
\hline Comportamento & 46,3 & 26,9 & 13,4 & 8,9 & 4,5 \\
\hline Comunicação & 52,2 & 32,8 & 6,0 & 6,0 & 3,0 \\
\hline Integridade cutâneo-mucosa & 47,8 & 40,3 & 8,9 & 1,5 & 1,5 \\
\hline Nutrição e hidratação & 50,7 & 28,4 & 19,4 & 1,5 & 0 \\
\hline Sinais vitais & 98,5 & 1,5 & 0 & 0 & 0 \\
\hline Oxigenação & 98,5 & 1,5 & 0 & 0 & 0 \\
\hline Terapêutica & 100 & 0 & 0 & 0 & 0 \\
\hline
\end{tabular}


Neste estudo evidenciou-se que grande maioria dos idosos possuía algum déficit relacionado ao indicador cuidado corporal, estando os escores distribuídos segundo os valores: $25,4 \%$ autossuficiente; $20,9 \%$ requer supervisão de enfermagem na realização do cuidado corporal e conforto; $13,4 \%$ requer orientação e supervisão de enfermagem ao acompanhante para auxílio na higiene oral, higiene íntima, banho de chuveiro e medidas de conforto; $32,8 \%$ requer auxílio da enfermagem na higiene oral, higiene íntima, banho de chuveiro e medidas de conforto; e 7,5\% requer assistência efetiva da enfermagem para o cuidado corporal e medidas de conforto devido à restrição no leito.

A educação em saúde - definida no instrumento como a habilidade do paciente/família em receber e aceitar orientações sobre autocuidado - foi o segundo indicador mais afetado nos idosos estudados. Sua distribuição segundo os escores foi: $29,9 \%$ orientações de enfermagem ao paciente/família sobre autocuidado com pronta compreensão e aceitação das informações recebidas; $29,9 \%$ orientações de enfermagem ao paciente/família sobre autocuidado com dificuldades de compreensão, mas com pronta aceitação das informações recebidas; $19,4 \%$ orientações de enfermagem ao paciente/família sobre autocuidado com pronta compreensão, mas certa resistência às informações recebidas; $11,9 \%$ orientações de enfermagem ao paciente/família sobre autocuidado com pronta compreensão, mas elevada resistência às informações recebidas; e $8,9 \%$ orientações de enfermagem ao paciente/família sobre autocuidado com pronta compreensão, mas sem aceitação das informações recebidas.

A locomoção, por sua vez, é o terceiro indicador mais afetado. Apenas 37,3\% dos idosos residentes conseguem locomover-se de forma autossuficiente. As frequências dos escores que seguem são: $28,4 \%$ requer orientação e supervisão de enfermagem ao acompanhante para auxílio no uso de artefatos (órteses, próteses, muletas, bengalas, cadeiras de rodas, andadores); $14,9 \%$ requer encorajamento e supervisão da enfermagem para a deambulação; $11,9 \%$ requer o auxílio da enfermagem no uso de artefatos para a deambulação; e 7,5\% requer assistência efetiva de enfermagem para locomoção devido à restrição no leito.

No indicador eliminações, observou-se um índice elevado de idosos que demandam algum tipo de atenção da enfermagem para satisfazer de forma segura essa necessidade. Apenas 35,8\% dos idosos conseguem fazêla de forma autossuficiente, ao passo que $9 \%$ requer supervisão e controle pela enfermagem das eliminações; $46,2 \%$ requer orientação e supervisão de enfermagem ao acompanhante para auxílio no uso de comadre, papagaio, troca de fraldas, absorventes e outros, e controle, pela enfermagem, das eliminações; e $9 \%$ requer auxílio e controle pela enfermagem no uso de comadre, papagaio, troca de fraldas, absorventes e outros. Constatou-se que nenhum dos residentes demanda assistência efetiva de enfermagem, ou seja, são totalmente dependentes da enfermagem, para manipulação e controle de cateteres, drenos, dispositivo para incontinência urinária ou estomas.

Quanto à motilidade constatou-se que é realizada de forma autossuficiente por apenas $16,4 \%$ dos idosos estudados, ao passo que $83,6 \%$ possuem alguma necessidade especial que requer estímulo, supervisão, orientação ou auxílio efetivo da enfermagem para movimentação de um ou mais segmentos corporais.

Em posse da identificação das necessidades humanas básicas afetadas é possível a determinação do grau de dependência de enfermagem apresentada por esses sujeitos. De acordo com a Teoria das Necessidades Humanas Básicas, quanto à natureza, o grau de dependência pode ser total - está implícita a extensão, pois significa que a enfermagem tem que fazer pelo ser humano tudo aquilo que este não tem condições de fazer por si, seja qual for a causa; ou parcial - os cuidados de enfermagem podem situar-se em termos de ajuda, orientação, supervisão e encaminhamento( ${ }^{(10)}$.

A análise da distribuição dos sujeitos de acordo com seus níveis de dependência dos cuidados de enfermagem, estabelecido segundo os indicadores de saúde avaliados, permite verificar o predomínio do nível de dependência parcial, seguido da independência e da dependência total. 
Tabela 3: Distribuição dos indicadores segundo nível de dependência de cuidados de enfermagem. Maceió-AL, 2011.

\begin{tabular}{cccc}
\hline Indicadores & Independência & Dependência parcial & Dependência total \\
\hline Estado mental ou nível de consciência & 35,8 & 64,2 & 0 \\
Oxigenação & 98,5 & 1,5 & 0 \\
Sinais vitais & 98,5 & 1,5 & 0 \\
Nutrição e hidratação & 50,8 & 49,2 & 0 \\
Motilidade & 53,7 & 41,8 & 4,5 \\
Locomoção & 37,3 & 55,2 & 7,5 \\
Cuidado corporal & 25,4 & 67,1 & 7,5 \\
Eliminações & 35,8 & 64,2 & 0 \\
Terapêutica & 100 & 0 & 0 \\
Educação a saúde & 29,9 & 61,2 & 8,9 \\
Comportamento & 46,3 & 49,2 & 4,5 \\
Comunicação & 52,2 & 44,8 & 3,0 \\
Integridade cutâneo-mucosa & 47,8 & 50,7 & 1,5 \\
\hline
\end{tabular}

Seis indicadores apresentaram maior quantitativo de idosos avaliados com o escore um, caracterizando a independência, são estes: oxigenação, sinais vitais, nutrição e hidratação, motilidade, terapêutica e comunicação. Sete indicadores apresentaram maior quantitativo de idosos avaliados com escores equivalentes a dois, três e quatro, tendo sido classificados, portanto, no nível de dependência parcial. Os indicadores são: estado mental ou nível de consciência, locomoção, cuidado corporal, eliminações, educação a saúde, comportamento e integridade cutâneo-mucosa (Tabela 3). Ressalta-se que em nenhum indicador predominou o escore cinco, embora se observe poucos (porém significativos) casos de dependência total.
Ainda segundo a Teoria, as necessidades expostas através dos indicadores de cuidado, definidas como psicobiológicas, psicossociais e psicoespirituais, podem ser classificadas quanto ao nível de dependência. As mesmas, apresentadas na forma de quadro, permitem uma fácil visualização das relações entre necessidades demandadas e nível de dependência de cuidados de enfermagem, colaborando para uma compreensão sistemática dessas relações colaborando para organização do serviço (Quadro 1). É importante esclarecer que as necessidades psicoespirituais não foram avaliadas nessa pesquisa devido à complexidade que envolve o tema e a inexistência, dentro do sistema de classificação de paciente, de elementos que subsidiassem sua avaliação.

Quadro 1: Distribuição dos níveis de cuidados de enfermagem de acordo com a classificação das necessidades humanas básicas. Maceió-AL, 2011.

\begin{tabular}{|c|c|c|c|}
\hline \multirow{2}{*}{$\begin{array}{c}\text { Níveis de cuidados } \\
\text { de enfermagem }\end{array}$} & \multicolumn{2}{|c|}{ Classificação das necessidades humanas básicas } \\
\cline { 2 - 4 } Independência & $\begin{array}{c}\text { Oxigenação, sinais vitais, nutrição e } \\
\text { hidrataçăo, motilidade e terapêutica. }\end{array}$ & Comunicação. & Psicossociais \\
\hline Dependência parcial & $\begin{array}{c}\text { Locomoção, cuidado corporal, } \\
\text { eliminações, educação à saúde e } \\
\text { integridade cutâneo-mucosa. }\end{array}$ & $\begin{array}{c}\text { Estado mental ou nível de } \\
\text { consciência e comportamento. }\end{array}$ & \multirow{2}{*}{ Não avaliadas. } \\
\hline Dependência total & - & - & \\
\hline
\end{tabular}

\section{DISCUSSÃO}

A população da ILPI estudada, à época da coleta, era formada principalmente por homens (59,9\%). Este resultado é similar ao encontrado em estudos que investigam os perfil de idosos hospitalizados, em que a população masculina é maior que a feminina(13-14). Este dado, no entanto, difere da característica geral apresentada nas ILPI do estado de Alagoas, cuja população se constitui majoritariamente por mulheres $(53,7 \%)^{(7)}$.
Entre os dados de caracterização dos sujeitos, destaca-se para esta discussão a faixa etária elevada (acima de 75 anos) dos residentes. Segundo Censo Demográfico realizado em 2010, os idosos constituem $10,8 \%$ da população brasileira, uma parcela significativa e crescente quando comparada ao Censo anterior (realizado no ano 2000), em que a população idosa era de $8,6 \%$. Na região nordeste do país, no entanto, houve uma diminuição no percentual de idosos na população, que passou de 14,8\% em 2000 para 10,2\% em 2010. Alagoas seguiu a tendência nacional de aumento demográfico da 
população idosa passando de $7,2 \%$ no ano 2000 para $8,9 \%$ em $2010^{(1)}$.

O envelhecimento da população idosa é atribuído a um aumento do número de idosos nas faixas etárias mais elevadas (acima de 75 anos), fato este condizente com os dados levantados neste estudo, que mostra uma média de idade igual há 76,8 anos entre os residentes (sendo a média feminina $=79,7$ anos e a masculina $=73,9$ anos), observando-se um envelhecimento significativo destes sujeitos.

No que trata ao estado civil observa-se que a maioria dos residentes são solteiros (58,2\%). Esses valores diferem dos encontrados em um estudo envolvendo idosos assistidos por uma equipe de saúde da família, cenário em que $45,3 \%$ dos idosos relataram ser casados, $44 \%$ são viúvos, 9,3\% são desquitados/divorciados e apenas $1,3 \%$ se declara solteiro ${ }^{(15)}$. Este diferença tornase interessante, pois nos permite pensar que a condição de estar se relacionando e convivendo com alguém confere uma possibilidade maior dos sujeitos manteremse residindo em domicílio. Assim, o medo ou sensação de solidão, somado a outros fatores (como dificuldades financeiras, despreparo familiar em atender as necessidades dos idosos e a própria situação de abandono enfrentada pelos idosos), são elementos que podem levar os idosos a residirem em ILPI.

Quanto à escolaridade observa-se que um número elevado de sujeitos não haviam sido alfabetizados, fato este condizente com o último levantamento do IBGE no estado, que afirma que apenas $5,97 \%$ dos idosos no estado de Alagoas são alfabetizados ${ }^{(1)}$. Entre as principais implicações advindas com o analfabetismo para que o idoso mantenha seu autocuidado refere-se à dificuldade para a administração correta de medicações e a compreensão de orientações referentes ao uso de objetos e aparatos pessoais e domésticos.

No que diz respeito à necessidade de cuidados de enfermagem pelos idosos, o que mais se evidenciou foi à demanda de cuidados mínimos e intermediários. Valores semelhantes foram observados em estudos realizados junto a idosos assistidos em unidade hospitalar, quando se observou que o nível de cuidados de enfermagem mais demandado era o intermediário (52,2\%), seguido por cuidados mínimos $(35,4 \%)$ e semi-intensivos $(12,4 \%)^{(16)}$. Uma terceira pesquisa realizada em hospital de prontosocorro, tendo como sujeitos paciente idosos e não idosos, revelou que o nível de dependência mais evidente era o intermediário, seguido por cuidados mínimos e semi-intensivos, agravando-se o quadro de dependência com o aumentar da faixa etária ${ }^{(14)}$.

Comparando-se estes resultados com nosso estudo, nota-se um decréscimo nos índices percentuais de cuidados intermediários e semi-intensivos, e um aumento no índice de cuidados mínimos, o que nos leva a inferir que, dentro de uma ILPI existam idosos exigindo uma atenção diminuída no que se refere à prestação de cuidados de enfermagem. Os indicadores também comprovam a inexistência de pacientes com cuidados intensivos de enfermagem, fato supostamente esperado, pois, sempre que ocorre agravamento do quadro dos idosos, estes são transferidos para a unidade hospitalar onde recebem a devida assistência.

No que diz respeito aos indicadores avaliados de forma individual, o cuidado corporal, indicador com maior índice de alteração nos escores, constitui-se como um desafio as ILPI, visto que requer um quantitativo maior de profissionais capacitados para desenvolvê-los. Este indicador - também bastante alterado em pesquisa junto a idosos hospitalizados(16) - aponta uma característica dependente em maior ou menor escala dos idosos institucionalizados, denotando uma atenção especial da enfermagem com relação ao dimensionamento de pessoal, visto que interfere diretamente na qualidade da assistência de enfermagem prestada, pois promove a segurança do paciente, a qualidade da assistência e, consequentemente, a otimização da aplicação dos recursos em saúde ${ }^{(17)}$. Uma das características mais importantes do dimensionamento de pessoal de enfermagem é justamente o fato do mesmo ser fundamentado no perfil dos pacientes, neste caso o idoso(16). $^{(16)}$

A educação a saúde foi o segundo indicador mais alterado na coleta de dados, configurando-se como um importante elemento a ser considerado na organização do trabalho da equipe de saúde da instituição. Destaca-se que a educação para o autocuidado só pode ocorrer quando há interação entre os profissionais e os clientes sob a égide daquele saber-prática. Aos profissionais, cabe o compromisso de realizar um trabalho educativo voltado para promoção do autocuidado junto ao idoso, aos cuidadores, à família e à sociedade.

A locomoção, por sua vez, é o terceiro indicador mais afetado durante $o$ estudo. A dependência para locomoção também foi observada junto a idosos 
hospitalizados ${ }^{(14,16)}$, constatando-se em um dos estudos que apenas 15,3\% locomovem-se de forma autossuficiente $^{(14)}$. Embora uma diminuição da capacidade de locomoção seja esperada junto aos idosos, é fundamental avaliar-se que nível de incapacidade está sendo apresentado por esses sujeitos, visto que a locomoção reduzida interfere diretamente, tanto no desenvolvimento das atividades diárias, quanto na própria autoestima dos mesmos.

No indicador eliminações, observou-se um índice elevado de idosos que querem algum tipo de atenção da enfermagem para satisfazer de forma segura essa necessidade. Essa necessidade afetada em nível elevado também foi observada junto a idosos hospitalizados, onde boa parte dos sujeitos estudados possuíam algum grau de dependência de enfermagem para satisfação desta necessidade ${ }^{(13-16)}$. Além de demandar uma atenção especial por parte da equipe de saúde devido aos riscos a saúde advindas com estas dificuldades, as mesmas podem apresentar-se como um sério agravante para os quadros clínicos de morbidade já apresentados pelos idosos.

A mobilidade física prejudicada é um dos diagnósticos que mais interferem na saúde do indivíduo. Vemos que os idosos são altamente susceptíveis a desenvolvê-la, visto que o processo de envelhecimento leva à alterações significativas relacionadas à musculatura, articulação e equilíbrio, fatores que interferem diretamente na mobilidade. Os valores encontrados a partir desta pesquisa são semelhantes aos encontrados em estudos com idosos hospitalizados - onde se evidencia que apenas $14,6 \%$ são autossuficientes - e no contexto da saúde da família - onde $90,7 \%$ dos idosos são diagnosticados com mobilidade física prejudicada ${ }^{(13,15)}$.

Este déficit comumente se relacionava a fatores como enfraquecimento músculo-esquelético, força e resistência muscular diminuídas e dano perceptual ou cognitivo(18). Em nossa pesquisa, embora não tenha sido visualizado o mesmo quantitativo absoluto (100\% dos casos) de deficiência na mobilidade, possibilitou a visualização do elevado grau de dificuldade dos idosos para movimentarem-se e manterem suas mobilidades conservadas.

\section{CONCLUSÕES}

Os enfermeiros devem estar atentos às alterações que acompanham o processo do envelhecimento, sabendo interpretá-las e distingui-las, avaliando a capacidade funcional e as necessidades apresentadas pelos idosos. No contexto das ILPI as necessidades que se encontram mais afetadas, levantadas segundo o Sistema de Classificação de Pacientes, são: cuidado corporal, educação à saúde, locomoção, eliminações e mobilidade. A elevada pontuação nestes indicadores reflete diretamente na presença indispensável dos trabalhadores de enfermagem em quantidade e qualidade suficientes a fim de que sejam supridas de forma adequada as necessidades dos sujeitos.

O resultado obtido com a avaliação dos indicadores cuidado corporal e educação à saúde, permitem comprovar a importância do papel educativo do enfermeiro e equipe de enfermagem a fim de se promover a saúde dos sujeitos. Demonstra também que o investimento em práticas educativas é extremamente válido, principalmente considerando os importantes benefícios proporcionados à instituição e aos idosos em decorrência do aprendizado e melhora do autocuidado.

A pesquisa revela que a avaliação do grau de dependência dos cuidados de enfermagem mais comumente observada é a dependência parcial para o cuidado, observada nos quesitos: locomoção, cuidado corporal, eliminações, educação à saúde, integridade cutâneo-mucosa, estado mental ou nível de consciência e comportamento. Este nível de dependência revela que os cuidados de enfermagem podem situar-se em termos de ajuda, orientação, supervisão e encaminhamento. A independência para o cuidado foi observada nos indicadores oxigenação, sinais vitais, nutrição e hidratação, motilidade, terapêutica e comunicação, refletindo a capacidade do idoso para o autocuidado.

Assim, fica clara a importância do enfermeiro na equipe da ILPI, pois além de prestar uma assistência integral e capacitada aos idosos institucionalizados, é capaz de mensurar as necessidades humanas e o nível de dependência de cuidados dos sujeitos em relação à enfermagem. Conhecendo a demanda, o enfermeiro pode participar do processo decisório dos serviços, melhorando a alocação de recursos humanos, materiais e tecnológicos.

Essa pesquisa traz, ainda, contribuições para a estruturação de uma assistência de enfermagem de qualidade, visto que possibilita um planejamento das intervenções da equipe de enfermagem e do dimensionamento de recursos humanos e materiais em 
uma ILPI, subsidiando uma assistência integral e de qualidade aos idosos institucionalizados.

\section{REFERÊNCIAS}

1. Instituto Brasileiro de Geografia e Estatística [Internet]. Brasília: Ministério do Planejamento, Orçamento e Gestação (BR) [cited 2011 dec 02]. Censo demográfico 2010. Available from: http://www.censo2010.ibge.gov.br/sinopse/index.php. 2. Coelho Filho JM. Saúde do Idoso. In: Rouquayrol MZ; Gurgel M. Epidemiologia e Saúde. 7.ed. Rio de Janeiro: Medbook, 2013; p. 401-446.

3. Fragoso V. Humanização dos cuidados a prestar ao idoso institucionalizado. Revista IGT na Rede [Internet]. 2008 [cited 2011 dec 02];5(8):51-61. Available from: http://www.igt.psc.br. 4. Bessa MEP, Silva MJ. Motivações para o ingresso dos idosos em instituições de longa permanência e processos adaptativos: um estudo de caso. Texto Contexto Enferm. [Internet]. 2008 [cited 2012 jan 21];17(2):258-65. Available from: http://www.scielo.br/pdf/tce/v17n2/06.pdf. 5. Michel T, Lenardt MH, Betiolli SE, Neu DKM. Significado atribuído pelos idosos à vivência em uma instituição de longa permanência: contribuições para o cuidado de enfermagem. Texto Contexto Enferm [Internet]. 2012 [cited $2012 \mathrm{dec}$ 13];21(3):495-504. Available from: http://www.scielo.br/pdf/tce/v21n3/v21n3a02.pdf. 6. Creutzberg M, Gongalves LHT. Acoplamento entre instituições de longa permanência para idosos e universidades. Rev. Eletr. Enf. [Internet]. 2011 [cited 2012 nov 04];13(4):620-8. Available from:

http://www.fen.ufg.br/fen revista/v13/n4/pdf/v13n4a05.pdf. 7. Melo IAF, Kubrusly ES, Peixoto Júnior AA. Perfil das instituições de longa permanência para idosos no Estado de Alagoas no período de 2007 a 2008*. Epidemiol. Serv. Saúde. 2011;20(1):75-83.

8. Felician AM, Santos SSC, Pelzer MT, Oliveira AMN, Pinho LB. Construção de ferramenta avaliativa direcionada às instituições de longa permanência para idosos: relato de experiência. Rev. Eletr. Enf. [Internet]. 2011 [cited 2012 mar 21];13(3):474-82. Available from: http://www.fen.ufg.br/revista/v13/n3/v13n3a13.htm. 9. Creutzberg M, Gonçalves LHT, Sobottka EA. Instituição de longa permanência para idosos: a imagem que permanece. Texto Contexto Enferm. [Internet]. 2008 [cited $2011 \mathrm{dec} 19$ ]; 17(2):273-9. Available from:

http://www.scielo.br/pdf/tce/v17n2/08.pdf.

10. Horta WA. Processo de Enfermagem. 1st ed. São Paulo: EPU; 1979.

11. Perroca MG, Gaidzinski RR. Sistema de classificação de pacientes: construção e validação de um instrumento. Rev. Esc. Enf. USP [Internet]. 1998 [cited 2012 mar 21];32(2):153-68. Available from:

http://www.ee.usp.br/reeusp/upload/pdf/430.pdf. 12. Perroca, MG. Instrumento de Classificação de Pacientes de Perroca: validação clínica [thesis] [Internet]. São Paulo: Escola de Enfermagem/USP; 2000. 168p.

13. Sales FM, Santos I. Perfil de idosos hospitalizados e nível de dependência de cuidados de enfermagem: identificação de necessidades. Texto Contexto Enferm. [Internet]. 2007 [cited 2012 mar 21];16(3):495-502. Available from:

http://www.scielo.br/pdf/tce/v16n3/a16v16n3.pdf. 14. Zimmermann LP, Magnago TSBS, Urbanetto JS, Greco PBT, Viero NC, Ceron MDS, et al. Avaliação do grau de dependência de cuidados de enfermagem dos pacientes internados em pronto-socorro. Rev. Enferm. UFSM [Internet]. 2011 [cited 2012 dec 12];1(2):153-63. Available from: http://cascavel.ufsm.br/revistas/ojs-

2.2.2/index.php/reufsm/article/view/2449/1659.

15. Bachion MM, Araújo LAOA. Programa saúde da família: perfil dos idosos assistidos por uma equipe. Rev. Bras. Enferm. 2004;57(5):586-90.

16. Urbanetto JS, Marco R, Carvalho SM, Creutzberg M, Oliveira KF, Magnago TBS. Grau de dependência de idosos hospitalizados conforme o sistema de classificação de pacientes. Rev. bras. enferm. [Internet]. 2012

[cited 2013 July 20]; 65(6): 950-954. Available from: http://www.scielo.br/pdf/reben/v65n6/a10v65n6.pdf. 17. Gil GP, Vituri DW, Haddad MCL, Vannuchi MTO, Moreno FN. Dimensionamento de pessoal de enfermagem e grau de dependência do paciente em um hospital universitário. Rev. Eletr. Enf. [Internet]. 2011 [cited 20 jul 2013];13(3):456-63. Available from: http://www.fen.ufg.br/revista/v13/n3/v13n3a11.htm. 18. Aveiro MC, Driusso P, Barham EJ, Pavarini SCl, Oishi J. Mobilidade e risco de quedas de população idosa da comunidade de São Carlos. Ciência \& Saúde Coletiva [Internet]. 2012; [cited 2013 jul 20];17(9):2481-2488. Available from: http://www.redalyc.org/articulo.oa?id=63023703023.

Artigo recebido em 23/05/2012. Aprovado para publicação em 18/07/2013. Artigo publicado em 30/09/2013. 